\title{
Altered regional homogeneity in patients with unilateral acute open-globe injury: a resting-state functional MRI study
}

This article was published in the following Dove Press journal:

Neuropsychiatric Disease and Treatment

I August 2016

Number of times this article has been viewed

\author{
Xin Huang ${ }^{1,2, *}$ \\ Hai-Jun $\mathrm{Li}^{3, *}$ \\ Lei Ye' \\ Ying Zhang' \\ Rong Wei ${ }^{1}$ \\ Yu-Lin Zhong' \\ De-Chang Peng ${ }^{3}$ \\ Yi Shao'
}

'Department of Ophthalmology, The First Affiliated Hospital of Nanchang University, Nanchang, ${ }^{2}$ Department of Ophthalmology, The First People's Hospital of Jiujiang City, Jiujiang,

${ }^{3}$ Department of Radiology, The First Affiliated Hospital of Nanchang University, Nanchang, Jiangxi, People's Republic of China

*These authors contributed equally to this work
Correspondence: Yi Shao

Department of Ophthalmology, The First Affiliated Hospital of Nanchang University, 17 Yongwaizheng Street, Donghu District, Nanchang 330006, Jiangxi, People's Republic of China

Tel +86 79l 88692520

Email freebee99@163.com

De-Chang Peng

Department of Radiology, The First Affiliated Hospital of Nanchang University, 17 Yongwaizheng Street, Donghu District, Nanchang 330006, Jiangxi, People's Republic of China

$\mathrm{Tel}+8679188695132$

Email pengdcdoctor@163.com
Objective: To investigate the underlying regional homogeneity ( $\mathrm{ReHo}$ ) brain activity changes in patients with unilateral acute open-globe injury (OGI) and their relationship with their clinical features.

Patients and methods: In total, 18 patients with acute OGI (16 males and two females) and 18 healthy controls (HCs; 16 males and two females) closely matched in age, sex, and education status participated in the study. Each subject underwent a resting-state functional magnetic resonance imaging scan. The ReHo method was used to assess local features of spontaneous brain activity. Receiver-operating characteristic curve was used to distinguish OGIs from HCs. The nonparametric statistical analysis was used to explore the relationship between the observed mean ReHo values of the different brain areas and the behavioral performance.

Results: Compared with HCs, acute OGI patients had significantly increased ReHo values in the right cerebellum posterior lobe/lingual gyrus, left superior temporal gyrus/inferior frontal gyrus, left inferior frontal gyrus, left posterior cingulate cortex/precuneus, and left precentral operculum. However, there was no relationship between the observed mean ReHo values of the different brain areas and the behavioral performance.

Conclusion: Acute OGI may cause dysfunction in many brain regions, which may reflect the underlying pathologic mechanisms of acute vision loss in OGI patients.

Keywords: open-globe injury, ReHo, functional magnetic resonance imaging, resting state

\section{Introduction}

Becoming a worldwide public health issue, open-globe injury (OGI) is a common eye disease with sharp decline in vision. The incident rate of ocular trauma is expected to be $1.6 \% \pm 0.2 \%$ in North China. ${ }^{1}$ Clinically, ocular trauma is mainly divided into OGIs and closed-globe injuries. OGIs occur more often than closed-globe injuries. ${ }^{2}$ OGI leads to serious visual impairment and is often associated with corneal injury and iris prolapse, ${ }^{3}$ retinal detachment, ${ }^{4}$ glaucoma, ${ }^{5}$ and endophthalmitis. ${ }^{6}$ Currently, surgery is the main treatment for OGI. ${ }^{7}$ OGI causes serious damage to vision, even blindness in some OGI patients. ${ }^{8}$

At present, computed tomography (CT) and B-scan ultrasonography are important clinical tests for diagnosis of OGI. CT is a noninvasive imaging diagnostic method that can provide useful information to the diagnosis of OGI. ${ }^{9,10}$ However, CT can only diagnose the integrity of the eyeball; hence, it is insufficient to provide guidance for clinical treatment. ${ }^{11}$ Compared with CT, B-scan ultrasonography is an effective measurement for OGI. ${ }^{12} \mathrm{~B}$-scan ultrasonography can identify retinal detachments and retinal lesions in OGI patients, ${ }^{13}$ which is beneficial for the treatment of OGI. Moreover, B-scan ultrasonography 
can evaluate the prognosis of OGI. The abovementioned inspection methods focus merely on the ocular trauma in OGI. However, other visual systems, including the connecting pathways and the visual cortex, are often overlooked. Optic nerve atrophic diseases can lead to abnormal visual pathways and visual cortex, such as optic neurities. ${ }^{14}$ Clinically, we found that despite successful emergency surgeries in some acute OGI patients, their vision continued to decrease. The mechanisms of optic neuropathy and cerebral functional changes caused by OGI should be further explored.

Resting-state functional magnetic resonance imaging (fMRI) is a functional brain imaging technique that can evaluate intrinsic brain activity in subjects at rest. ${ }^{15}$ The regional homogeneity (ReHo) method is widely used to determine local synchronization of spontaneous fMRI signals and provides useful information on brain activities. ${ }^{16,17}$ Previous studies have demonstrated that ReHo has high test-retest reliability and neurobiological relevance and it could serve as a neuroimaging marker to investigate the human brain function. ${ }^{18,19}$ The ReHo method has been successfully deployed to investigate pathological mechanisms of neural diseases such as optic neuritis, ${ }^{20}$ Parkinson's disease, ${ }^{21}$ and sleep deprivation. ${ }^{22}$ However, it has not yet been used to explore the pathophysiological changes in OGI. Many studies focused on the mechanism of eye damage in acute OGI patients. OGI may be accompanied by impaired visual pathways and changes in activities of the cerebral cortex. This study was designed to evaluate regional spontaneous brain activities in patients with OGI and their relationship with behavioral performances.

\section{Patients and methods Subjects}

A total of 18 acute OGI patients (16 males and two females, with nine right eyes and nine left eyes of injured eye) were recruited from the Department of Ophthalmology, The First Affiliated Hospital of Nanchang University Hospital. They were diagnosed with unilateral acute OGI based on the following criteria: 1) severe ocular trauma in unilateral eye, 2) acute vision loss after ocular trauma, 3) low intraocular pressure in injured eye, 4) cornea and scleral rupture, 5) orbital CT or orbital MRI showing incomplete eyeball wall in injured eye, and 6) contralateral eye best-corrected vision $\geq 1.0$.

Subjects were excluded from the study if they had the following conditions: 1) anterior segment diseases before the injury (such as glaucoma, cataract, strabismus, and pterygium), 2) other ocular neuropathy (such as infection, inflammation and ischemia diseases, hereditary optic neuropathy, demyelinating diseases, ischemic optic neuropathy, vascular lesions, toxic lesions, and intraocular placeholder lesions), 3) mental nervous system disorders and cerebral infarction diseases such as systemic disorders, 4) diabetes and cardiovascular diseases, and 5) addictions (eg, drugs or alcohol).

Eighteen healthy controls (HCs; 16 males and two females) with similar age, sex, and education status were also recruited in this study. HCs were recruited from healthy volunteers among the citizens of Nanchang, Jiangxi Province, People's Republic of China. All HCs met the following criteria: 1) no abnormalities in brain structure with brain MRI (such as cerebral hemorrhage, cerebral infarction, cerebrovascular malformation, and a brain tumor), 2) no eye disease and naked eye or corrected visual acuity (VA) $>1.0,3$ ) no mental disorders (such as depression disorders, anxiety disorders, and Alzheimer's disease), and 4) no MRI scanning contraindications (such as cardiac pacemaker and other metal devices).

This study was authorized by the First Affiliated Hospital of Nanchang University Hospital's ethics committee. All research methods followed the Declaration of Helsinki and conformed to the principles of medical ethics. All subjects participated voluntarily and were informed of the purposes, methods, and the potential risks, and all subjects signed an informed consent form.

\section{MRI parameters}

MRI scanning was performed on a 3-T MR scanner (Trio, Siemens, Germany). High-resolution T1-weighted images were acquired as described previously. ${ }^{18}$ The T1 parameters (repetition time $=1,900 \mathrm{~ms}$, echo time $=2.26 \mathrm{~ms}$, thickness $=1.0 \mathrm{~mm}$, gap $=0.5 \mathrm{~mm}$, acquisition matrix $=256 \times 256$, field of view $=250 \times 250 \mathrm{~mm}^{2}$, and flip angle $=9^{\circ}$ ) and 240 functional image parameters (repetition time $=2,000 \mathrm{~ms}$, echo time $=30 \mathrm{~ms}$, thickness $=4.0 \mathrm{~mm}$, gap $=1.2 \mathrm{~mm}$, acquisition matrix $=64 \times 64$, flip angle $=90^{\circ}$, field of view $=220 \times 220 \mathrm{~mm}$, and 30 axial slices with Gradient-Recalled Echo-Planar Imaging pulse sequence) covering the whole brain were obtained.

\section{fMRI data analysis}

All the functional data were prefiltered with MRIcro (www. $\underline{\text { MRIcro.com) }}$ ) and preprocessed using SPM8 (http://www.fil. ion.ucl.ac.uk/spm) and DPARSF (http://rfmri.org/DPARSF), after slice timing, head motion correction, spatial normalization, smooth with a Gaussian kernel of $6 \times 6 \times 6 \mathrm{~mm}^{3}$ full-width at half-maximum. First, participants with head motion $>1.5 \mathrm{~mm}$ or $1.5^{\circ}$ in any of the six parameters $(x, y, z)$ were excluded. Then the functional images were spatially normalized to the Montreal Neurological Institute (MNI) 
space using the standard EPI template and resampled at a resolution of $3 \times 3 \times 3 \mathrm{~mm}^{3}$. Finally, detrending and bandpass filtering $(0.01-0.08 \mathrm{~Hz})$ of the fMRI data were used to reduce physiological noise. Based on Kendall's coefficient of concordance (KCC), ReHo computation was performed with REST (http://www.resting-fmri.Sourceforge.net). Individual ReHo maps were generated by calculating the KCC of the time series of a given voxel with those of its nearest neighbors (26 voxels) in a voxel-wise manner with the formula ReHo = $\sum\left(R_{\mathrm{i}}\right)^{2}-\mathrm{n}\left(R^{-}\right) 2 K^{2}\left(\mathrm{n}^{3}-\mathrm{n}\right) / 12$, where ReHo is the KCC for a given voxel, ranging from 0 to 1 ; when the ranked time series is more consistent with its adjacent ones, the $\mathrm{KCC}$ value is more close to $1 . k$ is the voxel number among time series (in our study, $k=27$, including one given voxel that was located in the cubic center and its adjacent 26 voxels); $n$ is the number of ranks; $R_{\mathrm{i}}$ is the sum rank of the $i$ th time point, and $\overline{\mathrm{R}}=(\mathrm{n}+1) K / 2$ is the mean of the $R_{\mathrm{i}} \mathrm{s}$. The KCC value was referred to the central voxel among the cluster. The individual KCC-ReHo map was generated in a voxel-wise basis for all datasets. To reduce the effect of individual variations in the $\mathrm{KCC}$ value, ReHo maps were normalized to the averaged $\mathrm{KCC}$ of the whole brain.

\section{Statistical analysis}

A general linear model analysis was performed with the SPM8 toolkit to calculate the group differences in ReHo values between acute OGI patients and HCs. The significance level was set at $P<0.05$, with Gaussian random field theory corrected, minimum $z>2.3$.

\section{Brain behavior correlation analysis}

Based on the ReHo findings, the different brain regions between-groups were classified as regions of interest with the REST software. All clinical data of OGI patients were collected, including duration from onset of OGI to restingstate fMRI scan and best-corrected VA of both eyes in all subjects using logarithmic VA chart. The nonparametric statistical analysis was performed to evaluate the relationship between the mean ReHo value in different brain regions and the related clinical features in OGI group. $P<0.05$ was considered statistically significant.

\section{Clinical data analysis}

For behavioral performances, two-sample Student's $t$-test was used for continuous data. $P<0.05$ was considered statistically significant. The IBM SPSS software Version 20.0 statistical software (IBM Corporation, Armonk, NY, USA) was used for the statistical analyses.

\section{Results Demographics and visual measurements}

There were no obvious differences in weight $(P=0.970)$, age $(P=0.924)$, but significant differences in best-corrected VA-right $(P<0.001)$ and best-corrected VA-left $(P<0.001)$ were observed between patients with OGIs and HCs. Details are presented in Table 1.

\section{ReHo differences}

Compared with HCs, acute OGI patients had significantly increased ReHo values in the right cerebellum posterior lobe/ lingual gyrus, left superior temporal gyrus/inferior frontal gyrus, left inferior frontal gyrus, left posterior cingulate cortex (PCC)/precuneus, and left precentral operculum (Figure 1 and Table 2). The mean values of altered ReHo between patients with OGI and HCs are shown in Figure 2.

\section{Correlation analysis of ReHo values and clinical outcomes in OGI group}

In the acute OGI group, there was no relationship between the observed mean ReHo values of the different brain areas and the behavioral performance, as shown by the nonparametric statistics analysis $(P>0.05)$.

\section{Receiver-operating characteristic curve}

We obtained different ReHo values between the OGI and HC groups, which could be utilized as markers to separate OGI patients from HCs. To test this possibility, the mean ReHo values in different brain regions were used for analysis of the receiver-operating characteristic curves. In this study, the values of the area under the curve were the right cerebellum posterior lobe/lingual gyrus (0.969), left superior temporal

Table I Demographic information and clinical measures of OGI patients and $\mathrm{HCs}$

\begin{tabular}{|c|c|c|c|c|}
\hline Conditions & $\begin{array}{l}\text { OGI } \\
\text { patients }\end{array}$ & HCs & $t$ & $P$-values* \\
\hline Male/female & $16 / 2$ & $16 / 2$ & $\mathrm{~N} / \mathrm{A}$ & $>0.99$ \\
\hline Age (years) & $44.61 \pm 14.08$ & $44.17 \pm|3.8|$ & 0.096 & 0.924 \\
\hline Weight (kg) & $60.17 \pm 4.64$ & $60.11 \pm 4.10$ & 0.038 & 0.970 \\
\hline Handedness & I8R & $18 R$ & N/A & $>0.99$ \\
\hline $\begin{array}{l}\text { Duration from onset } \\
\text { of OGI to rs-fMRI } \\
\text { scan (hours) }\end{array}$ & $26.95 \pm 9.05$ & $\mathrm{~N} / \mathrm{A}$ & $\mathrm{N} / \mathrm{A}$ & $\mathrm{N} / \mathrm{A}$ \\
\hline $\begin{array}{l}\text { Best-corrected } \\
\text { VA-right }\end{array}$ & $0.58 \pm 0.60$ & $1.26 \pm 0.21$ & -4.595 & $<0.001$ \\
\hline $\begin{array}{l}\text { Best-corrected } \\
\text { VA-left }\end{array}$ & $0.65 \pm 0.55$ & $1.22 \pm 0.17$ & -4.273 & $<0.001$ \\
\hline
\end{tabular}

Notes: Data presented as number or mean \pm standard deviation. *Independent Student's $t$-tests comparing the two groups $(P<0.05)$.

Abbreviations: $\mathrm{OGI}$, open globe injury; $\mathrm{HC}$, healthy control; N/A, not applicable; $\mathrm{R}$, right; rs-fMRI, resting-state functional magnetic resonance imaging; VA, visual acuity. 


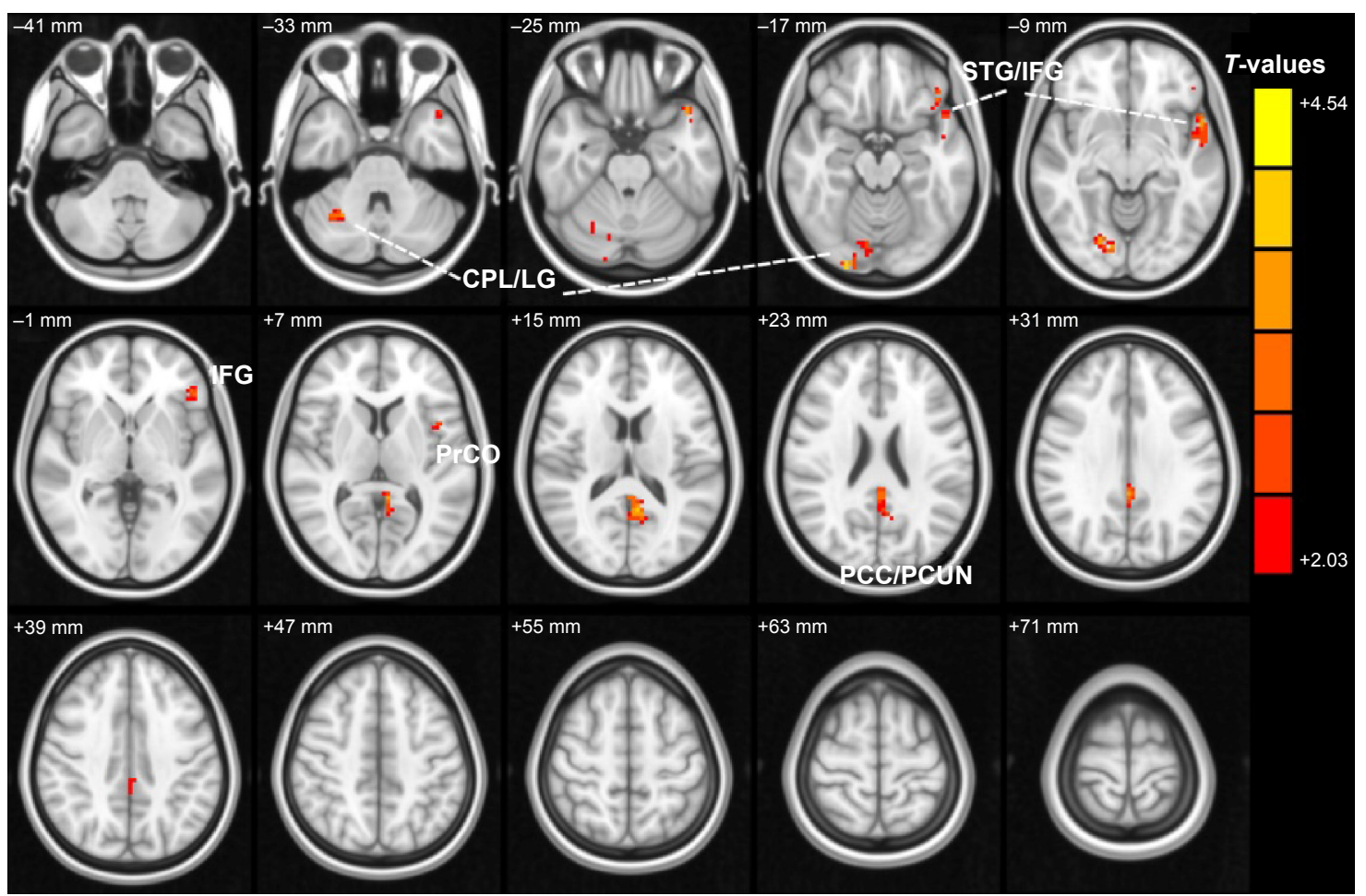

Figure I Spontaneous brain activity in the OGI and HC groups.

Notes: Significant differences in activity were observed for the right cerebellum posterior lobe/lingual gyrus, left superior temporal gyrus/inferior frontal gyrus, left inferior frontal gyrus, left posterior cingulate cortex/precuneus, and left precentral operculum. The red areas denote higher ReHo values. $P<0.05$ for multiple comparisons using Gaussian random field theory ( $z>2.3$, cluster-wise $P<0.05$ corrected).

Abbreviations: OGI, open globe injury; HC, healthy control; ReHo, regional homogeneity; CPL, cerebellum posterior lobe; LG, lingual gyrus; STG, superior temporal gyrus; IFG, inferior frontal gyrus; PCC, posterior cingulate cortex; PCUN, precuneus; PrCO, precentral operculum.

gyrus/inferior frontal gyrus (0.997), left inferior frontal gyrus (0.935), left PCC/precuneus (0.997), and left precentral operculum (0.923) (Figure 3).

\section{Discussion}

To our knowledge, this is the first study to evaluate the effects of acute OGI patients on resting-state brain activity using the ReHo technique and the relationship between fMRI and clinical results. Compared with HCs, acute OGI patients had significantly increased ReHo values in the right cerebellum posterior lobe/lingual gyrus, left superior temporal gyrus/ inferior frontal gyrus, left inferior frontal gyrus, left PCC/ precuneus, and left precentral operculum. The lingual gyrus located in the occipital lobe is involved in visual processing. ${ }^{23}$ A previous study reported high activities in the lingual area during visual encoding. ${ }^{24}$ Besides, the lingual gyrus has been suggested to control spatial memory ${ }^{25}$ and global shape processing. ${ }^{26}$ In our study, we found that OGI patients had significantly increased ReHo values in the lingual gyrus, indicating a stronger lingual gyrus activity than that in HCs. We speculated that OGI may lead to dysfunction of the lingual gyrus.

The superior temporal gyrus located in the temporal lobe is involved in auditory, ${ }^{27}$ language processing, ${ }^{28}$ and audiovisual encoding. ${ }^{29}$ A previous study demonstrated that

Table 2 Brain regions with significant differences in ReHo between OGI patients and HCs

\begin{tabular}{|c|c|c|c|c|c|c|}
\hline \multicolumn{4}{|l|}{ OGI patients and $\mathrm{HCs}$} & \multicolumn{3}{|c|}{ MNI coordinates } \\
\hline Brain areas & BA & T-values & $\begin{array}{l}\text { Peak } \\
\text { voxels }\end{array}$ & $x$ & $y$ & $\mathbf{z}$ \\
\hline Right cerebellum posterior lobe/lingual gyrus & 18 & 4.299 & 183 & 24 & -99 & -15 \\
\hline Left superior temporal gyrus/inferior frontal gyrus & $38,22,21$ & 3.893 & 119 & -45 & 33 & -3 \\
\hline Left inferior frontal gyrus & 47 & 3.284 & 13 & -42 & 30 & -15 \\
\hline Left posterior cingulate cortex/precuneus & $31,30,29,23$ & 4.516 & 163 & -6 & -54 & 12 \\
\hline Left precentral operculum & 44 & 2.674 & 14 & -45 & 9 & 6 \\
\hline
\end{tabular}

Note: The statistical threshold was set at voxel with $P<0.05$ for multiple comparisons using Gaussian random field theory ( $z>2.3$, cluster-wise $P<0.05$ corrected). Abbreviations: ReHo, regional homogeneity; OGI, open globe injury; HCs, healthy controls; MNI, Montreal Neurological Institute; BA, Brodmann area. 


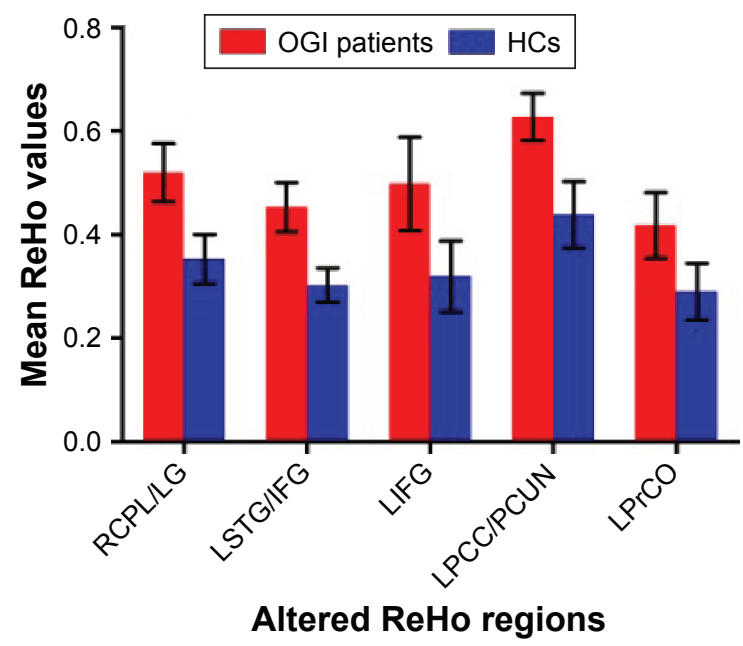

Figure 2 The mean of altered ReHo values between patients with OGls and HCs. Abbreviations: ReHo, regional homogeneity; OGl, open globe injury; HCs, healthy controls; RCPL, right cerebellum posterior lobe; LG, lingual gyrus; LSTG, left superior temporal gyrus; IFG, inferior frontal gyrus; LIFG, left inferior frontal gyrus; LPCC, left posterior cingulate cortex; PCUN, precuneus; LPrCO, left precentral operculum.

the superior temporal gyrus played an important role in the auditory-visual encoding. ${ }^{30}$ Additionally, it is responsible for complex visual stimuli processing, including facial movements and hand actions. ${ }^{31}$ In our study, we observed that OGI patients had significantly increased ReHo values in the left superior temporal gyrus, which may be related to the compensation of visual loss in OGI patients.

The PCC, located in the back of the cingulate cortex, is a central node in the default mode network. The PCC

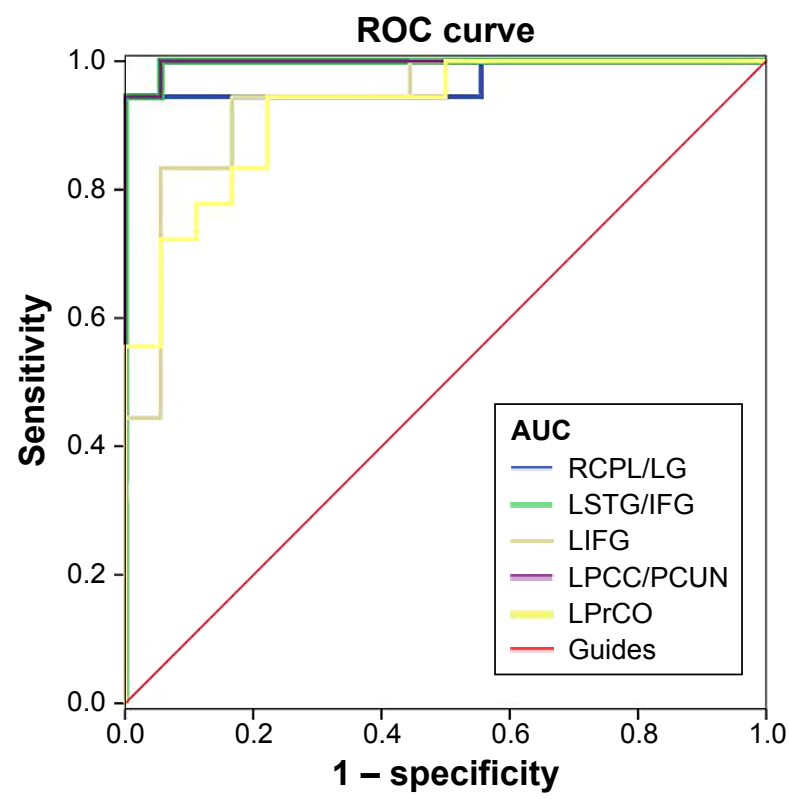

Figure $3 \mathrm{ROC}$ curve analysis of the mean ReHo values for altered brain regions. Note: The areas under the ROC curve (AUCs) for ReHo values: RCPL/LG (0.969), LSTG/IFG (0.997), LFG (0.935), LPCC/PCUN (0.997), and LPrCO (0.923).

Abbreviations: ROC, receiver-operating characteristic; ReHo, regional homogeneity; RCPL, right cerebellum posterior lobe; LG, lingual gyrus; LSTG, left superior temporal gyrus; IFG, inferior frontal gyrus; LIFG, left inferior frontal gyrus; LPCC, left posterior cingulate cortex; PCUN, precuneus; LPrCO, left precentral operculum. played an important role in cognitive. ${ }^{32}$ Moreover, the metabolic reduction in the PCC is seen in early Alzheimer's disease. ${ }^{33}$ Previous studies demonstrated the presence of altered cannabinoid receptor and GABA receptor in the PCC in schizophrenia patients. ${ }^{34,35}$ In our study, we found that patients with OGI showed higher ReHo values in the left PCC. Thus, we speculated that OGI may lead to the dysfunction of PCC.

The precuneus located on forward of the occipital lobe contributes to visuospatial ${ }^{36}$ and memory. ${ }^{37}$ Meanwhile, it is the core part of default-mode network. ${ }^{38}$ Moreover, previous studies have demonstrated the dysfunction of precuneus in many diseases such as Alzheimer disease, ${ }^{39}$ depression,,${ }^{40}$ and Schizophrenia. ${ }^{41}$ In our study, we found higher ReHo values in the precuneus in patients with OGI. We speculated that OGI may lead to dysfunction of the precuneus.

\section{Conclusion}

We found that patients with OGI patients had abnormal spontaneous activity in many brain regions, which may be related to the compensation of vision loss in acute OGI patients. These findings provided important information for the understanding of neural changes in unilateral OGI patients. However, there were some limitations to our study, such as the relatively small sample size. Moreover, the clinical characteristics were not strictly defined. We included both right eye and left eye injury patients, which might affect the ReHo findings. In future studies, we will distinguish these differences and measure brain function activity changes more accurately.

\section{Acknowledgments}

This study was supported by the National Natural Science Foundation of China (81400372). This was not an industrysupported study.

\section{Disclosure}

The authors report no conflicts of interest in this work.

\section{References}

1. Wang JD, Xu L, Wang YX, You QS, Zhang JS, Jonas JB. Prevalence and incidence of ocular trauma in North China: the Beijing eye study. Acta Ophthalmol. 2012;90(1):e61-e67.

2. Cao H, Li L, Zhang M. Epidemiology of patients hospitalized for ocular trauma in the Chaoshan region of China, 2001-2010. PLoS One. 2012; 7(10): 48377

3. Nawani N, Vazirani J, Ojha H, Sangwan VS. Conjunctival pedicle flap in management of open globe injury with corneal tissue loss. BMJ Case Rep. 2016;2016:bcr2015213703.

4. Stryjewski TP, Andreoli CM, Eliott D. Retinal detachment after open globe injury. Ophthalmology. 2014;121(1):327-333.

5. Osman EA. Glaucoma after open globe injury. Saudi J Ophthalmol. 2015;29(3):222-224 
6. Zhang Y, Zhang MN, Jiang CH, Yao Y, Zhang K. Endophthalmitis following open globe injury. Br J Ophthalmol. 2010;94(1):111-114.

7. Chorągiewicz T, Nowomiejska K, Wertejuk K, et al. Surgical treatment of open globe trauma complicated with the presence of an intraocular foreign body. Klin Oczna. 2015;117(1):5-8.

8. Ukponmwan CU, Momoh RO. Broomstick injuries to the eye; an emerging cause of blindness among children in Nigeria. Niger J Surg. 2015;21(1):13-17.

9. Hoffstetter P, Schreyer AG, Schreyer CI, et al. Multidetector CT (MD-CT) in the diagnosis of uncertain open globe injuries. Rofo. 2010; 182(2):151-154.

10. Arey ML, Mootha VV, Whittemore AR, Chason DP, Blomquist PH. Computed tomography in the diagnosis of occult open-globe injuries. Ophthalmology. 2007;114(8):1448-1452.

11. Allon G, Beiran I, Seider N, Blumenthal EZ. The role of computed tomography in the immediate workup of open globe injury. Eur J Ophthalmol. Epub 2016 Mar 30.

12. Berinstein DM, Gentile RC, Sidoti PA, et al. Ultrasound biomicroscopy in anterior ocular trauma. Ophthalmic Surg Lasers. 1997;28(3): 201-207.

13. Andreoli MT, Yiu G, Hart L, Andreoli CM. B-scan ultrasonography following open globe repair. Eye (Lond). 2014;28(4):381-385.

14. Huang X, Zhang Q, Hu PH, et al; fMRI Study Group AO. White and gray matter volume changes and correlation with visual evoked potential in patients with optic neuritis: a voxel-based morphometry study. Med Sci Monit. 2016;22:1115-1123.

15. Biswal BB. Resting state fMRI: a personal history. Neuroimage. 2012; 62(2):938-944

16. Zang Y, Jiang T, Lu Y, He Y, Tian L. Regional homogeneity approach to fMRI data analysis. Neuroimage. 2004;22(1):394-400.

17. Tononi G, McIntosh AR, Russell DP, Edelman GM. Functional clustering: identifying strongly interactive brain regions in neuroimaging data. Neuroimage. 1998;7(2):133-149.

18. Jiang L, Xu T, He Y, et al. Toward neurobiological characterization of functional homogeneity in the human cortex: regional variation, morphological association and functional covariance network organization. Brain Struct Funct. 2015;220(5):2485-2507.

19. Zuo XN, Xu T, Jiang L, et al. Toward reliable characterization of functional homogeneity in the human brain: preprocessing, scan duration, imaging resolution and computational space. Neuroimage. 2013;65: 374-386.

20. Shao Y, Cai FQ, Zhong YL, et al. Altered intrinsic regional spontaneous brain activity in patients with optic neuritis: a resting-state functional magnetic resonance imaging study. Neuropsychiatr Dis Treat. 2015; 11:3065-3073.

21. Li Y, Liang P, Jia X, Li K. Abnormal regional homogeneity in Parkinson's disease: a resting state fMRI study. Clin Radiol. 2016; 71(1):e28-e34.

22. Dai XJ, Gong HH, Wang YX, et al. Gender differences in brain regional homogeneity of healthy subjects after normal sleep and after sleep deprivation: a resting-state fMRI study. Sleep Med. 2012;13(6): 720-727.

23. Bogousslavsky J, Miklossy J, Deruaz JP, Assal G, Regli F. Lingual and fusiform gyri in visual processing: a clinico-pathologic study of superior altitudinal hemianopia. J Neurol Neurosurg Psychiatry. 1987;50(5): $607-614$.
24. Machielsen WC, Rombouts SA, Barkhof F, Scheltens P, Witter MP. FMRI of visual encoding: reproducibility of activation. Hum Brain Mapp. 2000;9(3):156-164.

25. Sulpizio V, Committeri G, Lambrey S, Berthoz A, Galati G. Selective role of lingual/parahippocampal gyrus and retrosplenial complex in spatial memory across viewpoint changes relative to the environmental reference frame. Behav Brain Res. 2013;242:62-75.

26. Mechelli A, Humphreys GW, Mayall K, Olson A, Price CJ. Differential effects of word length and visual contrast in the fusiform and lingual gyri during reading. Proc Biol Sci. 2000;267(1455):1909-1913.

27. Nourski KV, Brugge JF, Reale RA, et al. Coding of repetitive transients by auditory cortex on posterolateral superior temporal gyrus in humans: an intracranial electrophysiology study. J Neurophysiol. 2013; 109(5):1283-1295.

28. Bigler ED, Mortensen S, Neeley ES, et al. Superior temporal gyrus, language function, and autism. Dev Neuropsychol. 2007;31(2):217-238.

29. Uno T, Kawai K, Sakai K, et al. Dissociated roles of the inferior frontal gyrus and superior temporal sulcus in audiovisual processing: top-down and bottom-up mismatch detection. PLoS One. 2015;10(3): e0122580.

30. Reale RA, Calvert GA, Thesen T, et al. Auditory-visual processing represented in the human superior temporal gyrus. Neuroscience. 2007; 145(1):162-184.

31. Barraclough NE, Xiao D, Baker CI, Oram MW, Perrett DI. Integration of visual and auditory information by superior temporal sulcus neurons responsive to the sight of actions. J Cogn Neurosci. 2005;17(3): 377-391.

32. Leech R, Sharp DJ. The role of the posterior cingulate cortex in cognition and disease. Brain. 2014;137(pt 1):12-32.

33. Minoshima S, Giordani B, Berent S, Frey KA, Foster NL, Kuhl DE. Metabolic reduction in the posterior cingulate cortex in very early Alzheimer's disease. Ann Neurol. 1997;42(1):85-94.

34. Newell KA, Deng C, Huang XF. Increased cannabinoid receptor density in the posterior cingulate cortex in schizophrenia. Exp Brain Res. 2006;172(4):556-560.

35. Newell KA, Zavitsanou K, Jew SK, Huang XF. Alterations of muscarinic and GABA receptor binding in the posterior cingulate cortex in schizophrenia. Prog Neuropsychopharmacol Biol Psychiatry. 2007; 31(1):225-233.

36. Mahayana IT, Tcheang L, Chen CY, Juan CH, Muggleton NG. The precuneus and visuospatial attention in near and far space: a transcranial magnetic stimulation study. Brain Stimul. 2014;7(5):673-679.

37. Bonnì S, Veniero D, Mastropasqua C, et al. TMS evidence for a selective role of the precuneus in source memory retrieval. Behav Brain Res. 2015;282:70-75.

38. Utevsky AV, Smith DV, Huettel SA. Precuneus is a functional core of the default-mode network. J Neurosci. 2014;34(3):932-940.

39. Ikonomovic MD, Klunk WE, Abrahamson EE, et al. Precuneus amyloid burden is associated with reduced cholinergic activity in Alzheimer disease. Neurology. 2011;77(1):39-47.

40. Peng D, Liddle EB, Iwabuchi SJ, et al. Dissociated large-scale functional connectivity networks of the precuneus in medication-naïve first-episode depression. Psychiatry Res. 2015;232(3):250-256.

41. Faget-Agius C, Boyer L, Padovani R, et al. Schizophrenia with preserved insight is associated with increased perfusion of the precuneus. J Psychiatry Neurosci. 2012;37(5):297-304.
Neuropsychiatric Disease and Treatment

\section{Publish your work in this journal}

Neuropsychiatric Disease and Treatment is an international, peerreviewed journal of clinical therapeutics and pharmacology focusing on concise rapid reporting of clinical or pre-clinical studies on a range of neuropsychiatric and neurological disorders. This journal is indexed on PubMed Central, the 'PsycINFO' database and CAS,

\section{Dovepress}

and is the official journal of The International Neuropsychiatric Association (INA). The manuscript management system is completely online and includes a very quick and fair peer-review system, which is all easy to use. Visit http://www.dovepress.com/testimonials.php to read real quotes from published authors. 